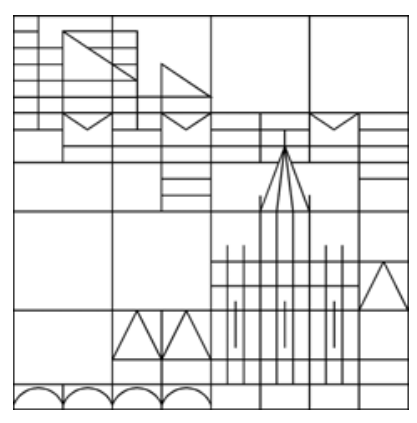

An inverse scattering problem for the time-dependent Maxwell equations : nonlinear optimization and model-order reduction

\author{
Roberta Mancini \\ Stefan Volkwein
}

Konstanzer Schriften in Mathematik

Nr. 297, Februar 2012

ISSN 1430-3558

Konstanzer Online-Publikations-System (KOPS)

URL: http://nbn-resolving.de/urn:nbn:de:bsz:352-184632

\footnotetext{
(C) Fachbereich Mathematik und Statistik

Universität Konstanz

Fach D 197, 78457 Konstanz, Germany
} 



\title{
An Inverse Scattering Problem for the Time-Dependent Maxwell Equations: Nonlinear Optimization and Model-Order Reduction
}

\author{
R. Mancini*, S. Volkwein \\ University of Constance, Department of Mathematics and Statistics, Germany
}

\begin{abstract}
SUMMARY
In this paper an inverse scattering problem for the time-dependent Maxwell curl equations is considered. This problem is formulated as an optimal control problem governed by partial differential equations. Firstorder necessary optimality conditions are discussed. For the numerical solution a gradient-based algorithm is applied and successfully tested for some numerical examples. Finally, model-order reduction based on proper orthogonal decomposition is utilized to derive a reduced-order model for the time-dependent Maxwell curl equations. Its applicability is tested with respect to different input frequencies. Copyright (c) 2010 John Wiley \& Sons, Ltd.
\end{abstract}

Received ...

KEY WORDS: Time-dependent Maxwell equations; inverse scattering; optimal control; nonlinear optimization; model reduction; proper orthogonal decomposition.

\section{INTRODUCTION}

Inverse scattering problems $[2,18,19,30,33]$ are very important in noninvasive imaging. In fact, because electromagnetic (EM) waves can penetrate in various media, where objects may be placed, the solution of inverse scattering problems allows to determine the presence and the properties of these objects hidden in the medium. Hence, one should deal with Maxwell's equations. They can be considered either in frequency or in time domain. In this paper the time domain version is used. In fact, data in time domain are richer in information because of the large spectrum of possible frequency of the incident field We focus on an unbounded domain, which arises in many applications, e.g., in mine detection [7]. Because of that, in the problem formulation we need to use an infinit radiation condition.

Optimal control problems for partial differential equations are often hard to tackle numerically so that the need for developing novel techniques emerges. One such technique is given by reducedorder methods. For a general overview we refer the reader to [1]. Recently the application of reduced-order models to optimal control problems has received an increasing amount of attention; see, e.g., $[14,25]$. The reduced-order approach is based on projecting the dynamical system onto subspaces consisting of basis elements that contain characteristics of the expected solution. This is in contrast to, e.g., finit element techniques, where the elements of the subspaces are uncorrelated to the physical properties of the system that they approximate. In this work we concentrate on the method of proper orthogonal decomposition (POD) $[15,35]$ as one of the reduced-order approaches. Since the solution of Maxwell equations - especially in three dimensions - has a significantl computational cost, we consider a POD Galerkin scheme for the FDTD scheme. The POD method is used here to reduce the EM fields For other model order reduction technique in the EM context,

*Correspondence to: Roberta Mancini, University of Constance, Department of Mathematics and Statistics, Universitätsstraße 10, D-78457 Konstanz, Germany 


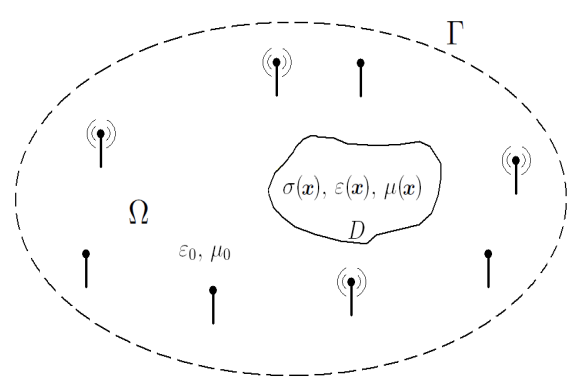

Figure 1. A cross section of scenario for an inverse scattering problem in the domain $\Omega$ and scatterer domain $D \subsetneq \Omega$. The antennas with the circles are the transmitting antennas in the incidence position, while the antennas without circles are the receiving ones.

refer to $[5,6,10,12]$. In [8] POD is applied for a f nite difference discretization using a staggered grid. For an error estimate we refer to [31]. The authors consider parabolic equations and use a f nite difference scheme as a high-dimensional discretization method. An error estimate is derived for the error between the solution to a parabolic equation and the associated POD Galerkin solution.

The paper is organized in the following manner: In Section 2 we formulate the inverse scattering problem as a nonlinear optimal control problem and present f rst-order necessary optimality conditions. The discretization of the optimality system is introduced in Section 3, where also numerical examples are shown. Section 4 is devoted on the proper orthogonal decomposition technique. Finally, we draw some conclusions in the last section.

\section{EM INVERSE SCATTERING PROBLEM IN OPTIMIZATION FRAMEWORK}

In this section, we formulate a time-domain electromagnetic (EM) inverse scattering problem in the framework of optimization with partial differential equations (PDEs) as constraints; [13, 34]. Our objective is to minimize a functional of EM f eld data misf $t$ including a regularization functional for the EM properties that are sought. To characterize the solution of the resulting optimization problem, we derive the corresponding f rst-order optimality system.

\subsection{Problem formulation}

Consider the scenario illustrated in Figure 1. This scenario consists of an unbounded domain $\Omega \subset \mathbb{R}^{3}$. The boundary of $\Omega$ - if it exists - is denoted by $\Gamma$. Moreover, let $T>0$ stand for the terminal time and $Q=(0, T) \times \Omega$. Let $D \subsetneq \Omega$ denote the scatterer domain and $Q_{D}=(0, T) \times D$. We suppose that $\Omega \backslash D$ has known EM properties, e.g., they can be the free space EM properties with electric permittivity $\varepsilon_{0}>0$, the magnetic permeability $\mu_{0}>0$ and conductivity $\sigma_{0}=0$. In the scatterer domain $D$ we assume that the EM properties $\varepsilon, \mu$, and $\sigma$ depend on the spatial variable $\boldsymbol{x} \in D$ and that they are different from those of the background medium. Moreover, we consider multiple antennas and receivers surrounding the scatterer domain $D$. The inverse scattering problem consists in the problem of reconstructing the EM properties $\boldsymbol{p}=(\varepsilon, \mu, \sigma)$ in the scatterer domain without accessing in it, knowing the EM f eld only in some points of the domain $\Omega \backslash D$. Throughout the paper we always assume that the scatterers have non-dispersive and isotropic EM properties (for detailed def nitions, see [11]).

The goal is to estimate the EM properties in the scatterer domain $D$ by utilizing the timedependent Maxwell curl equations as a mathematical model to predict the electrical and magnetic f eld $\boldsymbol{E}, \boldsymbol{H}: Q \rightarrow \mathbb{R}^{3}$ at $k_{\mathrm{m}}$ specif ed measurement points $\boldsymbol{x}_{k}^{\mathrm{m}} \in \Omega \backslash D, 1 \leq k \leq k_{\mathrm{m}}$. We suppose that measurement data is available at the points $\boldsymbol{x}_{k}^{\mathrm{m}}$. Since the EM properties $\boldsymbol{p}=(\varepsilon, \mu, \sigma)$ enter the Maxwell equations, we can identify these properties in $D$ in such a way that the corresponding f elds $\boldsymbol{E}=\boldsymbol{E}(\boldsymbol{p})$ and $\boldsymbol{H}=\boldsymbol{H}(\boldsymbol{p})$ are as close as possible to given data at the measurement points. In particular, we excite the Maxwell curl equations by $n_{\mathrm{i}}$ different sources in order to get 
$n_{\mathrm{i}}$ f eld variables $\left(\boldsymbol{E}_{n}, \boldsymbol{H}_{n}\right), 1 \leq n \leq n_{\mathrm{i}}$. We start with one incident wave in a certain position $\boldsymbol{x}_{1}^{\mathrm{i}} \in \Omega \backslash D$. Then, we compute the associated EM f elds $\left(\boldsymbol{E}_{1}, \boldsymbol{H}_{1}\right)$ in the $k_{\mathrm{m}}$ measurement points $\boldsymbol{x}_{i}^{\mathrm{m}}, 1 \leq k \leq k_{\mathrm{m}}$ over the time interval $[0, T]$. Then, we repeat the same procedure for the other $n_{\mathrm{i}}-1$ incidence points.

We introduce the parameter space

$$
\mathcal{P}=\boldsymbol{H}^{\mathbf{1}}(D) \cap \boldsymbol{L}^{\infty}(D),
$$

where $\boldsymbol{H}^{\mathbf{1}}(D)=H^{1}(D) \times H^{1}(D) \times H^{1}(D)$ and $\boldsymbol{L}^{\infty}(D)=L^{\infty}(D) \times L^{\infty}(D) \times L^{\infty}(D)$. The set of admissible parameters by

$$
\mathcal{P}_{\text {ad }}=\left\{(\varepsilon, \mu, \sigma) \in \mathcal{P} \mid \varepsilon_{a} \leq \varepsilon \leq \varepsilon_{b}, \mu_{a} \leq \mu \leq \mu_{b}, \sigma_{a} \leq \sigma \leq \sigma_{b} \text { almost everywhere in } D\right\}
$$

with positive lower and upper bounds $\varepsilon_{a}, \mu_{a}, \sigma_{a}, \varepsilon_{b}, \mu_{b}, \sigma_{b} \in L^{\infty}(D)$ satisfying $\varepsilon_{a} \leq \varepsilon_{b}, \mu_{a} \leq \mu_{b}$, $\sigma_{a} \leq \sigma_{b}$ in $D$ almost everywhere. Then, for any parameter vector $\boldsymbol{p} \in P_{\text {ad }}$ and for the $n$th incident wave $\left(1 \leq n \leq n_{\mathrm{i}}\right)$ the EM f elds $\left(\boldsymbol{E}_{n}, \boldsymbol{H}_{n}\right)$ satisfy the Maxwell curl equations (see, e.g., $\left.[11,28]\right)$

$$
\begin{array}{ll}
\nabla \times \boldsymbol{E}_{n}+\mu_{0} \frac{\partial \boldsymbol{H}_{n}}{\partial t}=0 & \text { in } Q \backslash Q_{D}, \\
\nabla \times \boldsymbol{E}_{n}+\mu \frac{\partial \boldsymbol{H}_{n}}{\partial t}=0 & \text { in } Q_{D}, \\
\nabla \times \boldsymbol{H}_{n}-\varepsilon_{0} \frac{\partial \boldsymbol{E}_{n}}{\partial t}-\boldsymbol{J}_{n}=0 & \text { in } Q \backslash Q_{D}, \\
\nabla \times \boldsymbol{H}_{n}-\varepsilon \frac{\partial \boldsymbol{E}_{n}}{\partial t}-\sigma \boldsymbol{E}_{n}=0 & \text { in } Q_{D} .
\end{array}
$$

Together with (1a) we pose the initial conditions

$$
\boldsymbol{E}_{n}(0, \cdot)=0, \quad \boldsymbol{H}_{n}(0, \cdot)=0 \quad \text { in } \Omega
$$

and the Sommerfeld radiation condition at inf nity

$$
\lim _{r \rightarrow \infty} r\left(\nabla \times\left(\begin{array}{c}
\boldsymbol{E}_{n} \\
\boldsymbol{H}_{n}
\end{array}\right)+\frac{1}{c_{0}} \mathfrak{n}_{r} \times \frac{\partial}{\partial t}\left(\begin{array}{c}
\boldsymbol{E}_{n} \\
\boldsymbol{H}_{n}
\end{array}\right)\right)=0 \quad \text { in } \Omega_{r}=\left\{\left.\boldsymbol{x} \in \mathbb{R}^{3}|| \boldsymbol{x}\right|_{2}<r\right\},
$$

where $k_{0}=\omega \sqrt{\mu_{0} \varepsilon_{0}}=2 \pi f \sqrt{\mu_{0} \varepsilon_{0}}$ and $\mathfrak{n}_{r}$ is the outward normal vector to $\partial \Omega_{r}$, the surface boundary of $\Omega_{r}$. In (1c) we use that $D \subsetneq \Omega_{r}$ for suff ciently large radius $r$. Finally, we def ne the f eld excitation. We consider a hard source [32], i.e., we assign a time dependent excitation function to a f eld component while the density current $\boldsymbol{J}_{n}$ is zero:

$$
\boldsymbol{H}_{n}\left(t, \boldsymbol{x}_{n}^{\mathrm{i}}\right)=\boldsymbol{f}_{n}(t) \quad \text { in }(0, T) .
$$

Remark 2.1

Let us mention that (1d) can be replaced by $\boldsymbol{E}_{n}\left(t, \boldsymbol{x}_{n}^{\mathrm{i}}\right)=\tilde{\boldsymbol{f}}_{n}(t)$ in $(0, T)$. A third possibility is to apply different current densities $\boldsymbol{J}_{n}$ in (1a) for $n=1, \ldots, n_{\mathrm{i}}$.

Assumption 1 (Existence and uniqueness for the Maxwell curl equations)

For any incident point $\boldsymbol{x}_{n}^{\mathrm{i}} \in \Omega \backslash D, 1 \leq n \leq n_{\mathrm{i}}$, and for any $\boldsymbol{p} \in \mathcal{P}_{\text {ad }}$ there exist unique felds $\boldsymbol{E}_{n}$ and $\boldsymbol{H}_{n}$ satisfying

$$
\boldsymbol{E}_{n}, \boldsymbol{H}_{n} \in C\left([0, T] ; \boldsymbol{H}^{\mathbf{1}}(\Omega) \cap \boldsymbol{C}(\bar{\Omega})\right) \cap H^{1}(0, T ; \boldsymbol{C}(\bar{\Omega}))=: y_{\mathrm{i}}
$$

for $n=1, \ldots, n_{\mathrm{i}}$, where $\boldsymbol{C}(\bar{\Omega})=C(\bar{\Omega}) \times C(\bar{\Omega}) \times C(\bar{\Omega})$.

Remark 2.2 1) The space $y_{i}$ is a Banach space endowed with the norm

$$
\|\boldsymbol{G}\|_{y_{i}}=\max _{t \in[0, T]}\left(\|\boldsymbol{G}(t)\|_{\boldsymbol{H}^{1}(\Omega)}+\|\boldsymbol{G}(t)\|_{\boldsymbol{C}(\bar{\Omega})}\right)+\left(\int_{0}^{1}\|\boldsymbol{G}(t)\|_{\boldsymbol{C}(\bar{\Omega})}^{2}+\|\dot{\boldsymbol{G}}(t)\|_{\boldsymbol{C}(\bar{\Omega})}^{2} \mathrm{~d} t\right)^{1 / 2}
$$


for $G \in y_{\mathrm{i}}$. We def ne the Banach spaces

$$
y=\underbrace{y_{i} \times \ldots \times y_{i}}_{2 n_{i}-\text { times }}, \quad x=y \times \mathcal{P}
$$

endowed with their natural product topology. Moreover let $X_{\text {ad }}=y \times \mathcal{P}_{\text {ad }} \subset X$.

2) For bounded domains, perfectly conducting boundary conditions and suitable smooth data it is shown in [20] that the Maxwell curl equations admits a unique solution.

3) If Assumption 1 holds true, we can def ne the solution operator $\mathcal{S}: \mathcal{P}_{\text {ad }} \rightarrow \mathcal{y}$, where

$$
(\boldsymbol{E}, \boldsymbol{H})=\left(\left(\boldsymbol{E}_{n}\right)_{n=1}^{n_{\mathrm{i}}},\left(\boldsymbol{H}_{n}\right)_{n=1}^{n_{\mathrm{i}}}\right)=\mathcal{S}(\boldsymbol{p}), \quad \boldsymbol{p}=(\varepsilon, \mu, \sigma) \in \mathcal{P}_{\mathrm{ad}},
$$

denotes the solution pairs to (1) for the $n_{i}$ incident waves. Due to the three bilinear terms $\mu \frac{\partial \boldsymbol{H}_{n}}{\partial t}, \varepsilon \frac{\partial \boldsymbol{E}_{n}}{\partial t}$ and $\sigma \boldsymbol{E}_{n}$ the operator $\mathcal{S}$ is nonlinear.

To write the inverse scattering problem as an optimization problem, we def ne a cost functional that penalizes the discrepancies between the measured data and the calculated felds. We aim at minimizing the misf $\mathrm{t}$ by varying the unknown EM properties in $D$ which def ne the optimization variables. Because of the ill-posedness of the inverse problem, we include a regularization term, $R(\boldsymbol{p})$. Thus we introduce the cost functional $\mathcal{J}: \mathcal{X} \rightarrow \mathbb{R}$ by

$$
\mathcal{J}(\boldsymbol{X})=\frac{1}{2} \sum_{n=1}^{n_{\mathrm{i}}} \sum_{k=1}^{k_{\mathrm{m}}} \int_{0}^{T}\left(\left|\boldsymbol{E}_{n k}(t)-\boldsymbol{E}_{n k}^{\mathrm{m}}(t)\right|_{2}^{2}+\eta_{0}^{2}\left|\boldsymbol{H}_{n k}(t)-\boldsymbol{H}_{n k}^{\mathrm{m}}(t)\right|_{2}^{2}\right) \mathrm{d} t+R(\boldsymbol{p}),
$$

where $\boldsymbol{X}=(\boldsymbol{E}, \boldsymbol{H}, \boldsymbol{p}) \in \mathcal{X}, \boldsymbol{E}=\left(\boldsymbol{E}_{1}, \ldots, \boldsymbol{E}_{n_{\mathrm{i}}}\right), \boldsymbol{H}=\left(\boldsymbol{H}_{1}, \ldots, \boldsymbol{H}_{n_{\mathrm{i}}}\right),|\cdot|_{2}$ denotes the Euclidean norm, $\eta_{0}>0$ is the characteristic impedance of free space, $\boldsymbol{E}_{n k}=\boldsymbol{E}_{n}\left(\cdot, \boldsymbol{x}_{k}^{\mathrm{m}}\right)$ and $\boldsymbol{H}_{n k}\left(\cdot, \boldsymbol{x}_{k}^{\mathrm{m}}\right)$ are the computed evaluated in the $k$ th measurement point for the $n$th incident wave f elds, whereas $\boldsymbol{E}_{n k}^{\mathrm{m}}$ and $\boldsymbol{H}_{n k}^{\mathrm{m}}$ are the associated measurement data. We consider the following regularization term

$$
\begin{aligned}
R(\boldsymbol{p})= & \frac{\beta}{2} \int_{D}|\nabla \varepsilon(\boldsymbol{x})|^{2}+|\nabla \sigma(\boldsymbol{x})|^{2}+|\nabla \mu(\boldsymbol{x})|^{2} \mathrm{~d} \boldsymbol{x} \\
& +\frac{\gamma}{2} \int_{D}|\varepsilon(\boldsymbol{x})-\bar{\varepsilon}(\boldsymbol{x})|^{2}+|\sigma(\boldsymbol{x})-\bar{\sigma}(\boldsymbol{x})|^{2}+|\mu(\boldsymbol{x})-\bar{\mu}(\boldsymbol{x})|^{2} \mathrm{~d} \boldsymbol{x}
\end{aligned}
$$

for $\boldsymbol{p}=(\varepsilon, \mu, \sigma) \in \mathcal{P}$. In this regularization functional we distinguish two different terms. The $\mathrm{f}$ rst term represents a f rst-order Tikhonov regularization scheme. Minimizing the functional $\mathcal{J}$ with this type of regularization means that we search the model parameters $\boldsymbol{p}$ in a smooth space with spatially slow varying functions. A result of this choice is that we obtain the edges of the reconstructed objects smeared out by diffusion. The other regularization term represents a zero-order Tikhonov regularization and corresponds to the requirement that $\varepsilon$ is as close as possible to a given nominal $\bar{\varepsilon}$, and similarly $\sigma$ is as close as possible to $\bar{\sigma}$, and $\mu$ is as close as possible to $\bar{\mu}$. This regularization term can be used even if we do not have a priori information with known $\bar{p}=(\bar{\varepsilon}, \bar{\mu}, \bar{\sigma})$. In this case we choose these values to be zero and the resulting regularization term penalizes large parameters values.

Now, the inverse scattering problem is expressed as the inf nite-dimensional, nonlinear optimization problem

$$
\min \mathcal{J}(\boldsymbol{X}) \quad \text { subject to (s.t.) } \quad \text { the pair } \boldsymbol{X}=(\boldsymbol{E}, \boldsymbol{H}, \boldsymbol{p}) \in \mathcal{X}_{\mathrm{ad}} \text { and }(\boldsymbol{E}, \boldsymbol{H})=\mathcal{S}(\boldsymbol{p}) .
$$

If Assumption 1 is satisf ed, we can introduce the reduced cost functional $\hat{\mathcal{J}}: \mathcal{P}_{\text {ad }} \rightarrow \mathbb{R}$ by

$$
\hat{\mathcal{J}}(\boldsymbol{p})=\mathcal{J}(\mathcal{S}(\boldsymbol{p}), \boldsymbol{p}), \quad \boldsymbol{p} \in \mathcal{P}_{\text {ad }}
$$

Then, $(\mathbf{P})$ can be equivalently expressed as the reduced problem

$$
\min \hat{\mathcal{J}}(\boldsymbol{p}) \quad \text { s.t. } \quad \boldsymbol{p} \in \mathcal{P}_{\mathrm{ad}} .
$$


Assumption 2 (Existence of optimal solutions)

Problem (P) admits at least one local optimal solution $\boldsymbol{X}^{*}=\left(\boldsymbol{E}^{*}, \boldsymbol{H}^{*}, \boldsymbol{p}^{*}\right) \in \mathcal{X}_{\mathrm{ad}}$. Moreover, $\boldsymbol{p}^{*}=\left(\varepsilon^{*}, \mu^{*}, \sigma^{*}\right)$ is an interior point of $\mathcal{P}_{\text {ad }}$, i.e., $\varepsilon_{a}<\varepsilon^{*}<\varepsilon_{b}, \mu_{a}<\mu^{*}<\mu_{b}$ and $\sigma_{a}<\sigma^{*}<\sigma_{b}$ in $D$ almost everywhere.

Remark 2.3 1) Let Assumption 2 hold. Then, $\boldsymbol{p}^{*}$ solves $(\hat{\mathbf{P}})$.

2) Suppose that Assumption 2 is satisf ed. Then, $\boldsymbol{p}^{*}$ is a local solution to

$$
\min \hat{\mathcal{J}}(\boldsymbol{p}) \quad \text { s.t. } \quad \boldsymbol{p} \in \mathcal{P} \text {. }
$$

In this case we can neglect the inequality constraints for the parameter $p$. Together with the associated state $\left(\boldsymbol{E}^{*}, \boldsymbol{H}^{*}\right)=\mathcal{S}\left(\boldsymbol{p}^{*}\right)$ the triple $\boldsymbol{X}^{*}=\left(\boldsymbol{E}^{*}, \boldsymbol{H}^{*}, \boldsymbol{p}^{*}\right)$ is a local solution $\boldsymbol{p}^{*}$ to the equality constrained problem

$$
\min \mathcal{J}(\boldsymbol{X}) \quad \text { s.t. } \quad \text { the pair } \boldsymbol{X}=(\boldsymbol{E}, \boldsymbol{H}, \boldsymbol{p}) \in \mathcal{X} \text { and }(\boldsymbol{E}, \boldsymbol{H})=\mathcal{S}(\boldsymbol{p}) . \quad\left(\mathbf{P}_{\text {eq }}\right)
$$

In the numerical experiments carried out in Section 3.2 we apply a gradient-type algorithm to $\left(\hat{\mathbf{P}}_{\text {eq }}\right)$ respectively $\left(\mathbf{P}_{\text {eq }}\right)$.

\subsection{First-order necessary optimality conditions}

Problem $\left(\hat{\mathbf{P}}_{\text {eq }}\right)$ is an inf nite-dimensional, constrained optimization problem. Thus, we apply techniques from PDE-optimization [13, 16, 34]. For this purpose we introduce the following Hilbert spaces

$$
z_{i}=L^{2}\left(0, T ; \boldsymbol{H}^{\mathbf{1}}(\Omega)\right) \cap H^{1}\left(0, T ; \boldsymbol{L}^{\mathbf{2}}(\Omega)\right), \quad z=\underbrace{z_{i} \times \ldots \times z_{i}}_{2 n_{i} \text {-times }}
$$

with the natural product topology and def ne the Lagrange functional $\mathcal{L}: X \times Z \rightarrow \mathbb{R}$ by

$$
\begin{aligned}
\mathcal{L}(\boldsymbol{X}, \boldsymbol{\Lambda})= & \mathcal{J}(\boldsymbol{X})+\sum_{n=1}^{n_{\mathrm{i}}} \int_{0}^{T} \int_{\Omega}\left(\boldsymbol{\lambda}_{n}^{E} \cdot\left(\nabla \times \boldsymbol{H}_{n}-\varepsilon \frac{\partial \boldsymbol{E}_{n}}{\partial t}-\sigma \boldsymbol{E}_{n}-\boldsymbol{J}_{n}\right)\right) \mathrm{d} \boldsymbol{x} \mathrm{d} t \\
& +\sum_{n=1}^{n_{\mathrm{i}}} \int_{0}^{T} \int_{\Omega}\left(\boldsymbol{\lambda}_{n}^{H} \cdot\left(\nabla \times \boldsymbol{E}_{n}+\mu \frac{\partial \boldsymbol{H}_{n}}{\partial t}\right)\right) \mathrm{d} \boldsymbol{x} \mathrm{d} t,
\end{aligned}
$$

where $\boldsymbol{X}=(\boldsymbol{E}, \boldsymbol{H}, \boldsymbol{p}) \in \mathcal{X}, \boldsymbol{\Lambda}=\left(\boldsymbol{\lambda}^{E}, \boldsymbol{\lambda}^{H}\right) \in \mathcal{Z}$ with $\boldsymbol{\lambda}_{E}=\left(\boldsymbol{\lambda}_{1}^{E}, \ldots, \boldsymbol{\lambda}_{n_{\mathrm{i}}}^{E}\right), \boldsymbol{\lambda}^{H}=\left(\boldsymbol{\lambda}_{1}^{H}, \ldots, \boldsymbol{\lambda}_{n_{\mathrm{i}}}^{H}\right)$ and $\boldsymbol{\lambda}_{i}^{E}, \boldsymbol{\lambda}_{i}^{H} \in Z_{\mathrm{i}}$ for $1 \leq i \leq n_{\mathrm{i}}$.

To solve the constrained minimization problem numerically, we want to make use of the frstorder optimality conditions. If one can ensure the existence of Lagrange multipliers [22], an optimal solution $\boldsymbol{X}^{*}$ to $\left(\mathrm{P}_{\text {eq }}\right)$ can be characterized by a stationary point of the Lagrange functional. Let Assumptions 1 and 2 hold. By $\boldsymbol{X}^{*}=\left(\boldsymbol{E}^{*}, \boldsymbol{H}^{*}, \boldsymbol{p}^{*}\right) \in X_{\text {ad }}$ we denote a local optimal solution to $\left(\mathbf{P}_{\text {eq }}\right)$. Moreover, let $\boldsymbol{Y}^{*}=\left(\boldsymbol{E}^{*}, \boldsymbol{H}^{*}\right)=\mathcal{S}\left(\boldsymbol{p}^{*}\right) \in \mathcal{y}$.

Assumption 3 (First-order necessary optimality conditions)

The Lagrangian $\mathcal{L}$ is continuously Fréchet-differentiable. Moreover, exists an associated pair of Lagrange multipliers $\boldsymbol{\Lambda}^{*}=\left(\boldsymbol{\lambda}^{E, *}, \boldsymbol{\lambda}^{H, *}\right) \in \mathcal{Z}$ satisfying the following frst-order necessary optimality conditions:

$$
\begin{array}{rlrl}
\frac{\partial \mathcal{L}}{\partial \boldsymbol{Y}}\left(\boldsymbol{X}^{*}, \boldsymbol{\Lambda}^{*}\right) \boldsymbol{Y} & =0 & & \text { for all } \boldsymbol{Y}=(\boldsymbol{E}, \boldsymbol{H}) \in \mathcal{y}, \\
\frac{\partial \mathcal{L}}{\partial \boldsymbol{p}}\left(\boldsymbol{X}^{*}, \boldsymbol{\Lambda}^{*}\right) \boldsymbol{p}=0 & & \text { for all } \boldsymbol{p} \in \mathcal{P},
\end{array}
$$

where $\frac{\partial \mathcal{L}}{\partial \boldsymbol{Y}}, \frac{\partial \mathcal{L}}{\partial \boldsymbol{p}}$ denote the Fréchet derivatives of the Lagrangian $\mathcal{L}$ with respect to $\boldsymbol{Y}$, and $\boldsymbol{p}$, respectively.

Remark 2.4

The existence of Lagrange multipliers can be ensured by constraint qualif cation conditions; see $[16,22,34]$, for instance. Due to (1c) the proof is not evident in our case. 
With Assumption 3 holding, we proceed formally by computing the directional derivatives of the Lagrange functional. For the details we refer the reader to the thesis [21].

From (5a) we infer that the pair $\Lambda=\left(\boldsymbol{\lambda}^{E, *}, \boldsymbol{\lambda}^{H, *}\right) \in \mathcal{Z}$ satisf es the following adjoint system

$$
\begin{array}{ll}
\nabla \times \boldsymbol{\lambda}_{n}^{E, *}-\mu_{0} \frac{\partial \boldsymbol{\lambda}_{n}^{H, *}}{\partial t}=0 & \text { in } Q \backslash Q_{D}, \\
\nabla \times \boldsymbol{\lambda}_{n}^{E, *}-\mu \frac{\partial \boldsymbol{\lambda}_{n}^{H, *}}{\partial t}+\eta_{0}^{2} \sum_{k=1}^{k_{\mathrm{m}}}\left(\boldsymbol{H}_{n}^{*}-\boldsymbol{H}_{n}^{\mathrm{m}}\right) \delta_{\boldsymbol{x}_{k}^{\mathrm{m}}}=0 & \text { in } Q_{D}, \\
\nabla \times \boldsymbol{\lambda}_{n}^{H, *}+\varepsilon_{0} \frac{\partial \boldsymbol{\lambda}_{n}^{E, *}}{\partial t}=0 & \text { in } Q \backslash Q_{D}, \\
\nabla \times \boldsymbol{\lambda}_{n}^{H, *}+\varepsilon \frac{\partial \boldsymbol{\lambda}_{n}^{E, *}}{\partial t}-\sigma \boldsymbol{\lambda}_{n}^{E, *}+\sum_{k=1}^{k_{\mathrm{m}}}\left(\boldsymbol{E}_{n}^{*}-\boldsymbol{E}_{n}^{\mathrm{m}}\right) \delta_{\boldsymbol{x}_{k}^{\mathrm{m}}}=0 & \text { in } Q_{D}, \\
\boldsymbol{\lambda}_{n}^{E, *}(T, \cdot)=0, \quad \begin{array}{l}
\boldsymbol{\lambda}_{n}^{H, *}(T, \cdot)=0 \\
\lim _{r \rightarrow \infty} r\left(\nabla \times\left(\begin{array}{l}
\boldsymbol{\lambda}_{n}^{E, *} \\
\boldsymbol{\lambda}_{n}^{H, *}
\end{array}\right)+\frac{1}{c_{0}} \mathfrak{n}_{r} \times \frac{\partial}{\partial t}\left(\begin{array}{c}
\boldsymbol{\lambda}_{n}^{E, *} \\
\boldsymbol{\lambda}_{n}^{H, *}
\end{array}\right)\right)=0
\end{array} & \text { in } \Omega_{r}=\left\{\left.\boldsymbol{x} \in \mathbb{R}^{3}|| \boldsymbol{x}\right|_{2}<r\right\}
\end{array}
$$

for every incidence $n \in\left\{1, \ldots, n_{\mathrm{i}}\right\}$. In (6) we denote by $\delta_{\boldsymbol{x}_{k}^{\mathrm{m}}}: C(\bar{D}) \rightarrow \mathbb{R}$ the Dirac delta mapping satisfying $\delta_{\boldsymbol{x}_{k}^{\mathrm{m}}}(\boldsymbol{x})=1$ if $\boldsymbol{x}=\boldsymbol{x}_{k}^{\mathrm{m}}$ holds. Otherwise, we have $\delta_{\boldsymbol{x}_{k}^{\mathrm{m}}}(\boldsymbol{x})=0$.

Let us have a closer look to system (6). Lagrange multipliers satisfy Maxwell-like equations, in which the signs of the time derivatives are opposite with respect to the standard curl equations. Moreover, we see that they satisfy terminal conditions and particular boundary conditions, in which there is a change in the time derivative. It can be shown that Lagrange multipliers $\boldsymbol{\lambda}_{n}^{E, *}$ and $\boldsymbol{\lambda}_{n}^{H, *}$ are special kind of EM f elds [21, 29]. In particular, they run backward in time, i.e., from $t=T$ to $t=0$. We remark also the fact that these particular f elds are excited by the discrepancies between the measurements and the predictions of the model in the $k_{\mathrm{m}}$ measurement points. If the model could exactly $\mathrm{ft}$ the scenario, i.e. the predictions of the model $\mathrm{ft}$ the measurements, the adjoint f elds would have zero sources.

From (5b) we deduce the following equations in the dual space $H^{1}(D)^{\prime}$ of $H^{1}(D)$

$$
\begin{aligned}
& \frac{\partial \mathcal{L}}{\partial \varepsilon}\left(\boldsymbol{X}^{*}, \boldsymbol{\Lambda}^{*}\right)=-\beta \Delta \varepsilon^{*}+\gamma\left(\varepsilon^{*}-\bar{\varepsilon}\right)-\sum_{n=1}^{n_{\mathrm{i}}} \int_{0}^{T} \frac{\partial \boldsymbol{E}_{n}^{*}}{\partial t} \cdot \boldsymbol{\lambda}_{n}^{E, *} \mathrm{~d} t=0, \\
& \frac{\partial \mathcal{L}}{\partial \mu}\left(\boldsymbol{X}^{*}, \boldsymbol{\Lambda}^{*}\right)=-\beta \Delta \mu^{*}+\gamma\left(\mu^{*}-\bar{\mu}\right)+\sum_{n=1}^{n_{\mathrm{i}}} \int_{0}^{T} \frac{\partial \boldsymbol{H}_{n}^{*}}{\partial t} \cdot \boldsymbol{\lambda}_{n}^{H, *} \mathrm{~d} t=0, \\
& \frac{\partial \mathcal{L}}{\partial \sigma}\left(\boldsymbol{X}^{*}, \boldsymbol{\Lambda}^{*}\right)=-\beta \Delta \sigma^{*}+\gamma\left(\sigma^{*}-\bar{\sigma}\right)-\sum_{n=1}^{n_{\mathrm{i}}} \int_{0}^{T} \boldsymbol{E}_{n}^{*} \cdot \boldsymbol{\lambda}_{n}^{E, *} \mathrm{~d} t=0 .
\end{aligned}
$$

It can be shown [13] that the gradient $\hat{\mathcal{J}}^{\prime}(\boldsymbol{p})$ of the reduced cost functional $\hat{\mathcal{J}}$ at a given parameter $\boldsymbol{p} \in \boldsymbol{H}^{\mathbf{1}}(D)$ is given by the following functional:

$$
\begin{aligned}
\hat{\mathcal{J}}^{\prime}(\boldsymbol{p})=( & -\beta \Delta \varepsilon+\gamma(\varepsilon-\bar{\varepsilon})-\sum_{n=1}^{n_{\mathrm{i}}} \int_{0}^{T} \frac{\partial \boldsymbol{E}_{n}}{\partial t} \cdot \boldsymbol{\lambda}_{n}^{E} \mathrm{~d} t, \\
& -\beta \Delta \mu+\gamma(\mu-\bar{\mu})+\sum_{n=1}^{n_{\mathrm{i}}} \int_{0}^{T} \frac{\partial \boldsymbol{H}_{n}}{\partial t} \cdot \boldsymbol{\lambda}_{n}^{H} \mathrm{~d} t, \\
& \left.-\beta \Delta \sigma+\gamma(\sigma-\bar{\sigma})-\sum_{n=1}^{n_{\mathrm{i}}} \int_{0}^{T} \boldsymbol{E}_{n} \cdot \boldsymbol{\lambda}_{n}^{E} \mathrm{~d} t\right): \boldsymbol{H}^{\mathbf{1}}(D) \rightarrow \mathbb{R}^{3},
\end{aligned}
$$

where $(\boldsymbol{E}, \boldsymbol{H})=\mathcal{S}(\boldsymbol{p})$ and $\boldsymbol{\Lambda} \in \mathcal{Z}$ solve (6) for $1 \leq n \leq n_{\mathrm{i}}$. Hence, we use these three equations in our gradient-based optimization method for updating the EM properties in the scatterer domain $D$. 


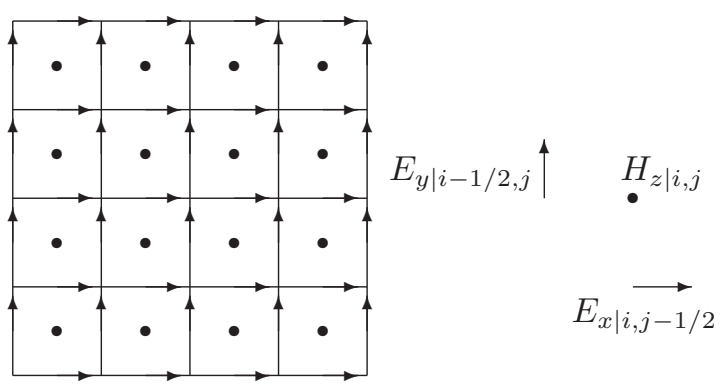

Figure 2. Spatial staggered grid arrangement for the $\mathrm{TE}_{z}$ wave.

For the update we have to solve the state equation (1) for the given parameter $\boldsymbol{p}$ and we have to determine the solution $\boldsymbol{\Lambda}$ to the adjoint equations.

\section{DISCRETIZATION OF THE OPTIMALITY SYSTEM}

There are several techniques for discretizing Maxwell equations (consider [17] for having a detailed overview of the most used techniques). We considered the f nite difference time domain (FDTD) algorithm. For details about the method, we refer the reader to [9, 32, 36]. For convergence results we refer the reader to [23], where the authors consider the Maxwell curl equtaions with simpler compared to the Sommerfeld radiation condition (1c) - boundary conditions.

\subsection{The FDTD scheme}

The discussion in Section 2 is valid in the general case of a three dimensional domain. Now we restrict ourselves to the two dimensional case, i.e., we do not consider variation in the $z$-direction (see [11, 32]). In this context, one can have two different types of waves: transverse electric $\left(\mathrm{TE}_{z}\right)$ and transverse magnetic $\left(\mathrm{TM}_{z}\right)$. We focus on the $\mathrm{TE}_{z}$ case, which has the felds components $E_{x}$, $E_{y}$ and $H_{z}: \boldsymbol{E}_{n}=\left(E_{x}, E_{y}, 0\right)^{\top}$ and $\boldsymbol{H}_{n}=\left(0,0, H_{z}\right)^{\top}$. For every incident wave $n$, they are coupled accordingly to the following system

$$
\begin{array}{llll}
\frac{\partial E_{x}}{\partial t}=\frac{1}{\varepsilon_{0}}\left(\frac{\partial H_{z}}{\partial y}-J_{s x}\right) & \text { in } Q \backslash Q_{D}, & \frac{\partial E_{x}}{\partial t}=\frac{1}{\varepsilon}\left(\frac{\partial H_{z}}{\partial y}-\left(J_{s x}+\sigma E_{x}\right)\right) & \text { in } Q_{D}, \\
\frac{\partial E_{y}}{\partial t}=\frac{1}{\varepsilon_{0}}\left(-\frac{\partial H_{z}}{\partial x}-J_{s y}\right) & \text { in } Q \backslash Q_{D}, & \frac{\partial E_{y}}{\partial t}=\frac{1}{\varepsilon}\left(-\frac{\partial H_{z}}{\partial x}-\left(J_{s y}+\sigma E_{y}\right)\right) & \text { in } Q_{D}, \\
\frac{\partial H_{z}}{\partial t}=\frac{1}{\mu_{0}}\left(\frac{\partial E_{x}}{\partial y}-\frac{\partial E_{y}}{\partial x}\right) & \text { in } Q \backslash Q_{D}, & \frac{\partial H_{z}}{\partial t}=\frac{1}{\mu}\left(\frac{\partial E_{x}}{\partial y}-\frac{\partial E_{y}}{\partial x}\right) & \text { in } Q_{D},
\end{array}
$$

where we skipped the index $n$.

We consider the FDTD method for discretizing the $\mathrm{TE}_{z}$ system. The FDTD scheme is characterized by the fact that the f eld components are considered in a staggered grid in space and in time. In our case, the spatial arrangement of the f elds component is like in Figure 2.

This implies that if we consider a space grid of dimension $M \times N$ for $H_{z}$, there will be $M \times(N+1)$ points for $E_{x}$ and $(M+1) \times N$ for $E_{y}$. For simplicity, we consider the case of an uniform grid, so we have the step sizes $\Delta x$ and $\Delta y$ on $x$ and $y$ directions, respectively. Moreover, magnetic and electric f elds are interleaved in time. Calling $\Delta t$ the time step and $N_{t}$ the number of time-steps, we evaluate $E_{x}$ and $E_{y}$ in the time instants $(m+1 / 2) \Delta t$ and $H_{z}$ in the time instants $m \Delta t$, with $m=0, \ldots, N_{t}$. It is worth noting that time step $\Delta t$ and step sizes $\Delta x$ and $\Delta y$ are related by the Courant condition $[9,32]$. Considering a square space grid, in which $h=\Delta x=\Delta y$, if one choose the time step $\Delta t$ then $\Delta$ should be taken in order to fulf ll $c \sqrt{2} \Delta t<h$, where $c$ is the speed in the vacuum. On the space-time grid, we consider $E_{x}(i \Delta x,(j-1 / 2) \Delta y ;(m+1 / 2) \Delta t) \approx$ $E_{x, i, j-1 / 2}^{m+1 / 2}, E_{y}((i-1 / 2) \Delta x, j \Delta y ;(m+1 / 2) \Delta t) \approx E_{y, i-1 / 2, j}^{m+1 / 2}$ and $H_{z}(i \Delta x, j \Delta y ; m \Delta t) \approx H_{z, i, j}^{m+1}$, 


$$
\begin{aligned}
& E_{y, I, J} \uparrow H_{z, I, J} \\
& \overrightarrow{E_{x, I, J}}
\end{aligned}
$$

Figure 3. Elementary cell for the $\mathrm{TE}_{z}$ case.

for $i=1, \ldots, M$ and $j=1, \ldots, N, m=0, \ldots, N_{t}$. Identifying the elementary cell by the couple of index $(I, J)$ as in Figure 3, the discrete system is given by

$$
\begin{gathered}
E_{x, I, J}^{m+1 / 2}=\frac{2 \varepsilon_{x, I, J}-\Delta t \sigma_{x, I, J}}{2 \varepsilon_{x, I, J}+\Delta t \sigma_{x, I, J}} E_{x, I, J}^{m-1 / 2}+\frac{2 \Delta t}{2 \varepsilon_{x, I, J}+\Delta t \sigma_{x, I, J}}\left(\frac{H_{z, I, J}^{m}-H_{z, I, J-1}^{m}}{\Delta x}-J_{s x, I, J}^{m}\right), \\
I=1, \ldots, M, J=2, \ldots, N \\
E_{y, I, J}^{m+1 / 2}=\frac{2 \varepsilon_{y, I, J}-\Delta t \sigma_{y, I, J}}{2 \varepsilon_{y, I, J}+\Delta t \sigma_{y, I, J}} E_{y, I, J}^{m-1 / 2}+\frac{2 \Delta t}{2 \varepsilon_{y, I, J}+\Delta t \sigma_{y, I, J}}\left(\frac{H_{z, I-1, J}^{m}-H_{z, I, J}^{m}}{\Delta x}-J_{s y, I, J}^{n}\right), \\
I=2, \ldots, M, J=1, \ldots, N, \\
H_{z, I, J}^{m+1}=H_{z, I, J}^{m}+\frac{\Delta t}{\mu_{I, J}}\left(\frac{E_{y, I, J}^{m+1 / 2}-E_{y, I+1, J}^{m+1 / 2}}{\Delta x}-\frac{E_{x, I, J+1}^{m+1 / 2}-E_{x, I, J}^{m+1 / 2}}{\Delta y}\right), \\
I=1, \ldots, M, J=1, \ldots, N \\
m=0, \ldots, N_{t} .
\end{gathered}
$$

Clearly, these equations provide an update for the inner grid points. This means that we need other equations for updating the boundary values of $E_{x}$ and $E_{y}$. Since we consider an unbounded domain, we update the felds in these points using some absorbing boundary condition (ABC) equations. A very popular $\mathrm{ABC}$ in the FDTD community is the perfectly matched layer (PML) [3]. In this approach, one surround the computational domain with a certain number of cells of an anisotropic, lossy medium, in which there are $\sigma_{x}, \sigma_{y}, \sigma_{x}^{*}$ and $\sigma_{y}^{*}$, where the latter 2 are the magnetic conductivities. Because of the anisotropy, in the PML region one need to split the the $H_{z}$ f eld component, i.e., one need to consider $H_{z x}$ and $H_{z y}$. Since next we would like to consider a model reduction technique, we consider another $\mathrm{ABC}$, in order to avoid the reduction of the split f eld components. We have used the second order Mur scheme [32], that follows from the EngquistMajda one-way wave equations which are

$$
\begin{array}{rlrl}
\frac{\partial E_{x}}{\partial t} & =c \frac{\partial E_{x}}{\partial y} & & \text { for the lower bound, } \\
\frac{\partial E_{x}}{\partial t} & =-c \frac{\partial E_{x}}{\partial y} & & \text { for the upper bound, } \\
\frac{\partial E_{y}}{\partial t}=c \frac{\partial E_{y}}{\partial x} & & \text { for the left bound, } \\
\frac{\partial E_{y}}{\partial t}=-c \frac{\partial E_{y}}{\partial x} & & \text { for the right bound, }
\end{array}
$$


where $c>0$ is the speed of the light in the vacuum. Using central differences, we get the secondorder Mur absorbing boundary conditions for the $E_{x}$ component [24]

$$
\begin{aligned}
E_{x, I, 1}^{m+1 / 2} & =-E_{x, I, 2}^{m-3 / 2}+\frac{c \Delta t-\Delta y}{c \Delta t+\Delta y}\left(E_{x, I, 2}^{m+1 / 2}+E_{x, I, 1}^{m-3 / 2}\right)+\frac{2 \Delta y}{c \Delta t+\Delta y}\left(E_{x, I, 1}^{m-1 / 2}+E_{x, I, 2}^{m-1 / 2}\right) \\
& +\frac{(c \Delta t)^{2}}{2 \Delta y(c \Delta t+\Delta y)}\left(E_{x, I+1,1}^{m-1 / 2}-2 E_{x, I, 1}^{m-1 / 2}+E_{x, I-1,1}^{m-1 / 2}+E_{x, I+1,2}^{m-1 / 2}-2 E_{x, I, 2}^{m-1 / 2}+E_{x, I-1,2}^{m-1 / 2}\right)
\end{aligned}
$$

for $I=2, \ldots, M-1$ and $m=0, \ldots, N_{t}$,

$$
\begin{aligned}
E_{x, I, N+1}^{m+1 / 2}= & -E_{x, I, N}^{m-3 / 2}+\frac{c \Delta t-\Delta y}{c \Delta t+\Delta y}\left(E_{x, I, N}^{m+1 / 2}+E_{x, I, N+1}^{m-3 / 2}\right)+\frac{2 \Delta y}{c \Delta t+\Delta y}\left(E_{x, I, N}^{m-1 / 2}+E_{x, I, N+1}^{m-1 / 2}\right) \\
& +\frac{(c \Delta t)^{2}}{2 \Delta y(c \Delta t+\Delta y)}\left(E_{x, I+1, N}^{m-1 / 2}-2 E_{x, I, N}^{m-1 / 2}+E_{x, I-1, N}^{m-1 / 2}\right) \\
& +\frac{(c \Delta t)^{2}}{2 \Delta y(c \Delta t+\Delta y)}\left(E_{x, I+1, N+1}^{m-1 / 2}-2 E_{x, I, N+1}^{m-1 / 2}+E_{x, I-1, N+1}^{m-1 / 2}\right)
\end{aligned}
$$

for $i=2, \ldots, M-1$ and $m=0, \ldots, N_{t}$,

$$
\begin{aligned}
& E_{x, 1,1}^{m+1 / 2}=E_{x, 1,2}^{m-1 / 2}+\frac{c \Delta t-\Delta y}{c \Delta t+\Delta y}\left(E_{x, 1,2}^{m+1 / 2}-E_{x, 1,1}^{m-1 / 2}\right), \\
& E_{x, M, 1}^{m+1 / 2}=E_{x, M, 2}^{m-1 / 2}+\frac{c \Delta t-\Delta y}{c \Delta t+\Delta y}\left(E_{x, M, 2}^{m+1 / 2}-E_{x, M, 1}^{m-1 / 2}\right), \\
& E_{x, 1, N+1}^{m+1 / 2}=E_{x, 1, N}^{m-1 / 2}+\frac{c \Delta t-\Delta y}{c \Delta t+\Delta y}\left(E_{x, 1, N}^{m+1 / 2}-E_{x, 1, N+1}^{m-1 / 2}\right), \\
& E_{x, M, N+1}^{m+1 / 2}=E_{x, M, N}^{m-1 / 2}+\frac{c \Delta t-\Delta y}{c \Delta t+\Delta y}\left(E_{x, M, N}^{m+1 / 2}-E_{x, M, N+1}^{m-1 / 2}\right),
\end{aligned}
$$

for $m=0, \ldots, N_{t}$.

Analogously, we get the equations for the feld component $E_{y}$.

Hence, we have all the equations we need for updating the f elds components in the computational grid: this means that we have a discrete scheme for solving the direct problem. We can combine the update equations in a compact matrix notation. For that purpose we def ne the vectors $\mathbf{E}_{x}^{m+1 / 2} \in$ $\mathbb{R}^{M(N+1)}, \mathbf{E}_{y}^{m+1 / 2} \in \mathbb{R}^{N(M+1)}, \mathbf{H}_{z}^{m+1} \in \mathbb{R}^{N M}$ for $m=0, \ldots, N_{t}$

$$
\begin{aligned}
\mathbf{E}_{x}^{m+1 / 2} & =\left(E_{x, 1,1}^{m+1 / 2}, \ldots, E_{x, M, 1}^{m+1 / 2}, E_{x, 1,2}^{m+1 / 2}, \ldots, E_{x, M, N}^{m+1 / 2}, E_{x, 1, N+1}^{m+1 / 2}, \ldots, E_{x, M, N+1}^{m+1 / 2}\right)^{\top} \\
\mathbf{E}_{y}^{m+1 / 2} & =\left(E_{y, 1,1}^{m+1 / 2}, \ldots, E_{y, M+1,1}^{m+1 / 2}, E_{y, 1,2}^{m+1 / 2}, \ldots, E_{y, M+1, N-1}^{m+1 / 2}, E_{y, 1, N}^{m+1 / 2}, \ldots, E_{y, M+1, N}^{m+1 / 2}\right)^{\top}, \\
\mathbf{H}_{z}^{m+1} & =\left(H_{z, 1,1}^{m+1}, \ldots, H_{z, M, 1}^{m+1}, H_{z, 1,2}^{m+1}, \ldots, H_{z, M, N-1}^{m+1}, H_{z, 1, N}^{m+1}, \ldots, H_{z, M, N}^{m+1}\right)^{\top} .
\end{aligned}
$$

Then, the new iterates $\mathbf{E}_{x}^{m+1 / 2}, \mathbf{E}_{y}^{m+1 / 2}$ and $\mathbf{H}_{z}^{m+1}$ are computed as follows: Solve the linear system

$$
\left(\begin{array}{cc}
\mathbf{M}_{x} & \mathbf{0} \\
\mathbf{0} & \mathbf{M}_{y}
\end{array}\right)\left(\begin{array}{l}
\mathbf{E}_{x}^{m+1 / 2} \\
\mathbf{E}_{y}^{m+1 / 2}
\end{array}\right)=\left(\begin{array}{cc}
\mathbf{A}_{E_{x}} & \mathbf{0} \\
\mathbf{0} & \mathbf{A}_{E_{y}}
\end{array}\right)\left(\begin{array}{l}
\mathbf{E}_{x}^{m-1 / 2} \\
\mathbf{E}_{y}^{m-1 / 2}
\end{array}\right)+\left(\begin{array}{c}
\mathbf{A}_{y} \mathbf{H}_{z}^{m}+\mathbf{J}_{s x}^{m} \\
\mathbf{A}_{x} \mathbf{H}_{z}^{m}+\mathbf{J}_{s y}^{m}
\end{array}\right)
$$

and set

$$
\mathbf{H}_{z}^{m+1}=\mathbf{H}_{z}^{m}+\mathbf{B}_{y} \mathbf{E}_{x}^{m+1 / 2}-\mathbf{B}_{x} \mathbf{E}_{y}^{m+1 / 2},
$$

where $\mathbf{M}_{x}, \mathbf{M}_{y}, \mathbf{A}_{E_{x}}, \mathbf{A}_{E_{y}}, \mathbf{A}_{x}, \mathbf{A}_{y}, \mathbf{B}_{x}, \mathbf{B}_{y}$ are matrices that contain the discretization coeff cients.

Next we consider now the discretization of the adjoint problem. Recall that the adjoint $f$ elds $\boldsymbol{\lambda}_{n}^{E}$ and $\boldsymbol{\lambda}_{n}^{H}$ are ingoing waves, with terminal conditions instead of initial conditions, i.e., they backpropagate in time starting from a f nal condition. In principle, we need a discretization scheme that runs with a decreasing time variable, in order to take into account this characteristic. In practice 
we can avoid this and using the same FDTD used for solving the direct problem, after performing the transformation $\tilde{t}=T-t$ and thus compute the modif ed Lagrange multipliers that are solution of the following system of equations

$$
\begin{array}{ll}
\nabla \times \tilde{\boldsymbol{\lambda}}_{n}^{E}+\mu_{0} \frac{\partial \tilde{\boldsymbol{\lambda}}_{n}^{H}}{\partial t}=0 & \text { in } Q \backslash Q_{D}, \\
\nabla \times \tilde{\boldsymbol{\lambda}}_{n}^{E}+\mu \frac{\partial \tilde{\boldsymbol{\lambda}}_{n}^{H}}{\partial t}+\eta_{0}^{2} \sum_{k=1}^{k_{\mathrm{m}}}\left(\tilde{\boldsymbol{H}}_{n}-\tilde{\boldsymbol{H}}_{n}^{\mathrm{m}}\right) \delta_{\boldsymbol{x}_{k}^{\mathrm{m}}}=0 & \text { in } Q_{D} \\
\nabla \times \tilde{\boldsymbol{\lambda}}_{n}^{H}+\varepsilon_{0} \frac{\partial \tilde{\boldsymbol{\lambda}}_{n}^{E}}{\partial t}=0 & \text { in } Q \backslash Q_{D}, \\
\nabla \times \tilde{\boldsymbol{\lambda}}_{n}^{H}+\varepsilon \frac{\partial \tilde{\boldsymbol{\lambda}}_{n}^{E}}{\partial t}-\sigma \tilde{\boldsymbol{\lambda}}_{n}^{E}+\sum_{k=1}^{k_{\mathrm{m}}}\left(\tilde{\boldsymbol{E}}_{n}-\tilde{\boldsymbol{E}}_{n}^{\mathrm{m}}\right) \delta_{\boldsymbol{x}_{k}^{m}}=0 & \text { in } Q_{D} .
\end{array}
$$

In fact, with the change of the time coordinate, the procedure used to compute $\tilde{\lambda}_{n}^{E}$ and $\tilde{\lambda}_{n}^{H}$ goes forward in time. At the end of the FDTD run for the modif ed Lagrange f elds, the adjoint f elds $\boldsymbol{\lambda}_{n}^{E}$ and $\lambda_{n}^{H}$ can be obtained reading the results from the f nal one to the $\mathrm{f}$ rst.

After solving forward and adjoint problem, we can approximate numerically the gradient components of the reduced cost functional $\hat{\mathcal{J}}(\boldsymbol{p})$ described in equations (7). The time derivatives are calculated using central differences approximation in time. We computed the time integration of the product terms using the trapezoidal rule. The terms related to the frst-order regularization require the computation of a Laplace operator $\Delta$. This operator is approximated using the f ve-point stencil rule. Having the gradient components, we can use them in an iterative scheme which updates the EM properties.

We have considered a steepest descent method. Hence, in the minimization problem of the functional $\hat{\mathcal{J}}$, one can f nd a descent direction $\boldsymbol{d}_{i}$ as $\boldsymbol{d}_{i}=-\hat{\mathcal{J}}^{\prime}\left(\boldsymbol{p}_{i}\right) \in \boldsymbol{H}^{\mathbf{1}}(D)^{\prime}$ for every iteration $i$ of the optimization scheme. Then, the update of the optimization variables is performed by $\boldsymbol{p}_{i+1}=\boldsymbol{p}_{i}+s_{i} \tilde{\boldsymbol{d}}_{i}$, where $s_{i}$ is a step length and $\tilde{\boldsymbol{d}}_{i}=\left(\tilde{d}_{i}^{\varepsilon}, \tilde{d}_{i}^{\mu}, \tilde{d}_{i}^{\sigma}\right) \in \boldsymbol{H}^{\mathbf{1}}(D)$ is the weak solution to the Neumann boundary value problem

$$
-\Delta \tilde{\boldsymbol{d}}_{i}+\tilde{\boldsymbol{d}}_{i}=\boldsymbol{d}_{i} \text { in } D, \quad \frac{\partial \tilde{\boldsymbol{d}}_{i}}{\partial \mathfrak{n}}=0 \text { on } \partial D .
$$

We considered also the BFGS for fnding at every step a new descent direction, but we did not observe remarkable improvements in the speed of the reconstruction algorithm.

The line search method gives us the step length $s_{i}$, i.e., it tells how much to move on the descent direction $d_{i}$. We considered a backtracking procedure that stopped once the Armijo condition was satisf ed (see, e.g., [26]).

\subsection{A numerical example}

In this subsection we consider a numerical example. We focus on the reconstruction of a dielectric scatterer. The algorithm is valid for multiple scatterers and/or magnetic and conductive scatterers, too.

In our experiment, the computational domain is a square whose side-length is three times larger than that of the scatterer square domain $D$. We assume the side-length of $D$ corresponding to a length of 20 cells. The number of time steps is set to $N_{t}=200$ and the time-step size is $\Delta t=10^{-6}$ seconds. The time domain is $(0, T)$ where $T=N_{t} \Delta t$ seconds. The spatial increment, $h$ is equal in the $x$ - and $y$-direction and it is set equal to $h=2 c \Delta t=5.99 \cdot 10^{2}$ meters, such that it satisf es the Courant limit [32]. The EM properties of the background are those one of the free space i.e., $\mu_{0}=4 \pi \cdot 10^{-7} \mathrm{H} / \mathrm{m}$ and $\varepsilon_{0}=8.854212 \cdot 10^{-12} \mathrm{~F} / \mathrm{m}$. The measurement points and the source points are placed in the region surrounding the domain $D$. We consider four different incidence points and eight measurement points, i.e. $n_{\mathrm{i}}=4$ and $k_{\mathrm{m}}=8$; see Figure 4 . To generate the incidence $\mathrm{f}$ elds, we 


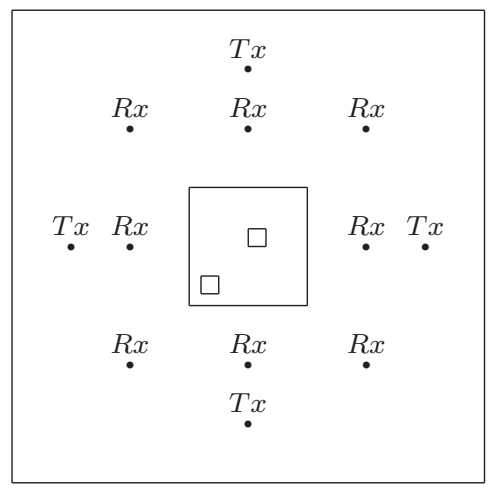

Figure 4. Picture of the position of transmitters $(T x)$ and receivers $(R x)$ in the domain. Two generic scatterers are depicted in the domain $D$.

have used hard sources [32]. This means that we set a variation of the magnetic f eld directly where the transmitters are placed. Hence, we need to specify $\boldsymbol{f}_{n}(t)$ in equation (1d) that in the $\mathrm{TE}_{z}$ case is $H_{z}=f(t)$, in the excitation points. As in [29], the excitation of the magnetic f eld was taken equal to the following

$$
H_{z}(t)= \begin{cases}\frac{1}{\eta_{0}}\left(\frac{3}{10} \sin \left(\frac{2 \pi t}{T_{1}}\right)+\sin \left(\frac{2 \pi t}{T_{2}}\right)\right), & 0 \leq t \leq T_{1}, \\ 0 & T_{1}<t,\end{cases}
$$

for every $n_{\mathrm{i}}$. Hence, we considered a truncated composition of sine wavefunctions with frequency of $20 \mathrm{kHz}$ and $50 \mathrm{kHz}$, respectively.

To evaluate our inversion algorithm, we have considered an hidden dielectric square with sidelength of ten cells and relative electrical permittivity $\varepsilon_{r}=3$. This square is placed such that the left lower corner has coordinate $(25,25)$ cell units in the computational domain. This square is depicted in Figure 5 with a eye-bird view. Hence, we solve the associated direct scattering problem and collect
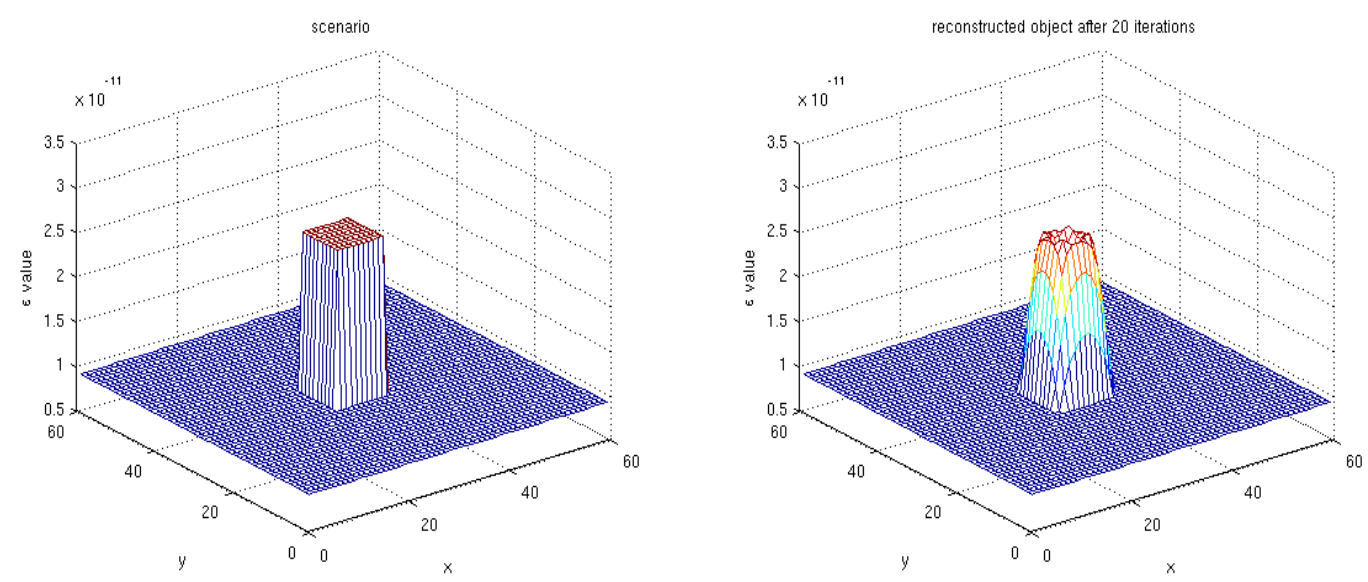

Figure 5. Simulated scenario (left plot) and reconstructed scatterer after 20 iterations (right plot)

the predictions at the receivers for all time-steps in $(0, T)$. We also built synthetic measurements: we take the felds obtained using the scatterer domain which we would like to reconstruct and we added noise (thus avoiding inverse crimes). We denote the measurements corresponding to the $n$th incidence feld and the $k$ th receiver as $\boldsymbol{E}_{n k}^{\mathrm{m}}$ and $\boldsymbol{H}_{n k}^{\mathrm{m}}$. We have used a white Gaussian noise such that the signal-to-noise ratio is $\mathrm{SNR}=10 \mathrm{~dB}$. For the two regularization terms we take $\beta=10^{-10}$ and $\gamma=10^{-10}$. This choice results from our experience. 
To discuss the convergence properties of the algorithm, we introduce a measure of the reconstruction error as follows

$$
\mathrm{ER}_{\varepsilon}=\sqrt{\frac{\sum_{l=1}^{L}\left(\varepsilon^{(l)}-\tilde{\varepsilon}^{(l)}\right)^{2}}{\sum_{l=1}^{L}\left(\varepsilon_{0}-\tilde{\varepsilon}^{(l)}\right)^{2}}},
$$

where $L=400$ is the number of cells of the scatterer domain $D$ and the tilde indicates the true value of the property. Of course, the same formula is valid for the reconstruction of magnetic and conductive objects. In Figure 6 the decay of the reconstructed error along the iterations is depicted.

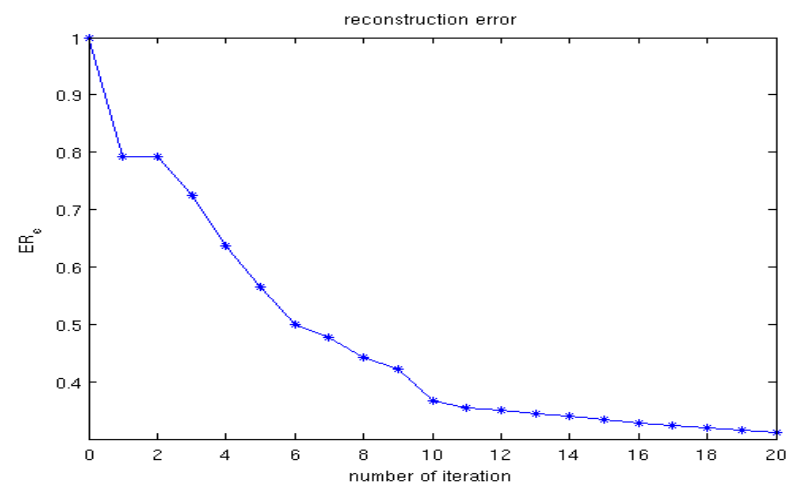

Figure 6. Reconstruction error along the iterations.

In the right plot of Figure 5, the reconstructed scatterer is presented. After 20 iterations, we obtain a good imaging of the true object. However, the reconstructed values are not constant in the square. In fact the edges tend to have greater values than those in the interior of the square. Instead of the true value $2.656 \cdot 10^{-11} \mathrm{~F} / \mathrm{m}$, we have as maximum value $2.695 \cdot 10^{-11} \mathrm{~F} / \mathrm{m}$ and in the interior we have a minimum value of $2.161 \cdot 10^{-11} \mathrm{~F} / \mathrm{m}$. This means that we have a maximum $\varepsilon_{r}=3.044$ and a minimum $\varepsilon_{r}=2.440$. That is, the optimization algorithm is more accurate in correspondence of the edges.

\section{A MODEL REDUCTION APPROACH: PROPER ORTHOGONAL DECOMPOSITION}

In this section we utilize the POD method to derive a reduced-order model for the FDTD scheme. The performance of the reduced-order approach is studied for different frequencies.

\subsection{The POD Galerkin scheme}

First we describe the POD basis construction. Dealing with a $\mathrm{TE}_{z}$ wave, we have three feld components, hence we build three basis set. First, we need to have the so-called snapshots matrices. To do so, we run an EM simulation using the FDTD method and we store the felds components in three matrices:

$$
\begin{aligned}
\mathbf{E}_{x} & =\left(\mathbf{E}_{x}^{1 / 2}, \ldots, \mathbf{E}_{x}^{N_{t}+1 / 2}\right) \in \mathbb{R}^{M(N+1) \times\left(N_{t}+1\right)}, \\
\mathbf{E}_{y} & =\left(\mathbf{E}_{y}^{1 / 2}, \ldots, \mathbf{E}_{y}^{N_{t}+1 / 2}\right) \in \mathbb{R}^{N(M+1) \times\left(N_{t}+1\right)}, \\
\mathbf{H}_{z} & =\left(\mathbf{H}_{z}^{1}, \ldots, \mathbf{H}_{z}^{N_{t}+1}\right) \in \mathbb{R}^{M N \times\left(N_{t}+1\right)} .
\end{aligned}
$$

Because of the staggered grid, the three matrices have different number of rows. Let $d_{x}$ denote the rank of $\mathbf{E}_{x}$. Then, $d_{x} \leq \min \left(M(N+1), N_{t}+1\right)$. Analogously, $d_{y}=\operatorname{rank} \mathbf{E}_{y} \leq \min (N(M+$ 1), $\left.N_{t}+1\right)$ and $d_{z}=\operatorname{rank} \mathbf{H}_{z} \leq \min \left(N M, N_{t}+1\right)$.

Let us focus on the feld component $\mathbf{E}_{x}$. The procedure is analogous for $\mathbf{E}_{y}$ and $\mathbf{H}_{z}$. In the POD approach, one would like to represent the columns $\mathbf{E}_{x}^{j}, 1 \leq j \leq N_{t}+1$, of the matrix $\mathbf{E}_{x}$, the socalled snapshots by $\ell_{x} \leq d_{x}$ vectors $\psi_{i} \in \mathbb{R}^{M(N+1)}, i=1, \ldots, \ell_{x}$, in an optimal way. The basis 
vectors $\psi_{i}$ are given by the following optimization problem:

$$
\begin{gathered}
\min _{\psi_{1}, \ldots, \psi_{\ell} \in \mathbb{R}^{d} x} \sum_{j=1}^{N_{t}+1} \alpha_{j}\left\|\mathbf{E}_{x}^{j}-\sum_{i=1}^{\ell_{x}}\left\langle\mathbf{E}_{x}^{j}, \psi_{i}\right\rangle_{\mathbf{W}} \psi_{i}\right\|_{\mathbf{W}}^{2} \\
\text { s.t. }\left\langle\psi_{i}, \psi_{j}\right\rangle_{\mathbf{W}}=\delta_{i j} \text { for } i, j=1, \ldots, \ell_{x},
\end{gathered}
$$

where $\langle\cdot, \cdot\rangle_{\mathbf{W}}$ denotes a weighted inner product with a symmetric, positive def nite matrix $\mathbf{W} \in$ $\mathbb{R}^{M(N+1) \times M(N+1)}$. We need to introduce this new inner product because the EM f elds are functions, hence the $L^{2}$ inner product is the natural product that we need. Introducing an opportune W matrix, we can approximate numerically this product. Moreover, we introduced weights $\alpha_{j}$, $j=1, \ldots, N_{t}+1$ for the time dependency of the EM felds. More precisely, the $\alpha_{j}$ are the trapezoidal weights for the numerical integration over $[0, T]$.

The problem means that one would like to minimize the difference, for every snapshot $\mathbf{E}_{x}^{j}$, between the snapshot itself and its projection in the subspace def ned by the basis vectors $\psi_{i}$, under the constraint of orthonormality property of the basis. Let us def ne $\mathbf{D}=\operatorname{diag}\left(\alpha_{1}, \ldots, \alpha_{N_{t}+1}\right)$.

Using the Lagrangian framework and considering the $\psi_{i}$ vectors as optimization variables, the optimality system is an $M(N+1) \times M(N+1)$ eigenvalue problem [15,35]

$$
\mathbf{Y} \mathbf{Y}^{\top} \psi_{i}=\lambda_{i} \psi_{i}, \quad i=1, \ldots, \ell_{x},
$$

with $\mathbf{Y}=\mathbf{W}^{1 / 2} \mathbf{E}_{x} \mathbf{D}^{1 / 2}$. We assume that the (real and nonnegative) eigenvalues are ordered as follows: $\lambda_{1} \geq \lambda_{2} \geq \ldots \geq \lambda_{d_{x}}>\lambda_{d_{x}+1}=\ldots=\lambda_{M(N+1)}=0$.

Remark 4.1 1) According to the singular values decomposition properties we can solve the equivalent $\left(N_{t}+1\right) \times\left(N_{t}+1\right)$ eigenvalue problem:

$$
\mathbf{Y}^{\top} \mathbf{Y} v_{i}=\lambda_{i} v_{i}, \quad i=1, \ldots, \ell_{x},
$$

Then, the $\psi_{i}$ 's are given by

$$
\psi_{i}=\frac{1}{\sqrt{\lambda_{i}}} \mathbf{Y} v_{i}, \quad i=1, \ldots, \ell_{x} .
$$

2) If $\left\{\psi_{i}\right\}_{i=1}^{\ell_{x}}$ denotes the computed POD basis of rank $\ell_{x}$, then we have the error formula

$$
\sum_{j=1}^{N_{t}+1} \alpha_{j}\left\|\mathbf{E}_{x}^{j}-\sum_{i=1}^{\ell_{x}}\left\langle\mathbf{E}_{x}^{j}, \psi_{i}\right\rangle_{\mathbf{W}} \psi_{i}\right\|_{\mathbf{W}}^{2}=\sum_{i=\ell+1}^{d_{x}} \lambda_{i}
$$

Thus, if the eigenvalues of $\mathbf{Y Y} \mathbf{Y}^{\top}$ decay rapidly, then $\sum_{i=\ell+1}^{d_{x}} \lambda_{i}$ is small.

Summarizing, for every f eld components we get a basis: $\Psi_{x} \in \mathbb{R}^{M(N+1) \times \ell_{x}}, \Psi_{y} \in \mathbb{R}^{N(M+1) \times \ell_{y}}$, $\Phi_{z} \in \mathbb{R}^{N M \times \ell_{z}}$ for the felds $E_{x}, E_{y}, H_{z}$, respectively.

The POD bases are used in the full discrete model (8) for a Galerkin ansatz:

$$
\mathbf{E}_{x}^{m+1 / 2} \approx \Psi_{x} \mathbf{P}_{x}^{m+1 / 2}, \quad \mathbf{E}_{y}^{m+1 / 2} \approx \Psi_{y} \mathbf{P}_{y}^{m+1 / 2}, \quad \mathbf{H}_{z}^{m} \approx \Phi_{z} \mathbf{P}_{z}^{m},
$$

where $\mathbf{P}_{x} \in \mathbb{R}^{\ell_{x}}, \mathbf{P}_{y} \in \mathbb{R}^{\ell_{y}}$ and $\mathbf{P}_{z} \in \mathbb{R}^{\ell_{z}}$ have to be determined. We apply a Galerkin projection projection for the full discrete model. Instead of (8) we arrive at

$$
\left(\begin{array}{cc}
\mathbf{M}_{x}^{\ell} & \mathbf{0} \\
\mathbf{0} & \mathbf{M}_{y}^{\ell}
\end{array}\right)\left(\begin{array}{c}
\mathbf{P}_{x}^{m+1 / 2} \\
\mathbf{P}_{y}^{m+1 / 2}
\end{array}\right)=\left(\begin{array}{cc}
\mathbf{A}_{E_{x}}^{\ell} & \mathbf{0} \\
\mathbf{0} & \mathbf{A}_{E_{y}}^{\ell}
\end{array}\right)\left(\begin{array}{l}
\mathbf{P}_{x}^{m-1 / 2} \\
\mathbf{P}_{y}^{m-1 / 2}
\end{array}\right)+\left(\begin{array}{c}
\mathbf{A}_{y}^{\ell} \mathbf{P}_{z}^{m}+\mathbf{W}_{x}^{\ell} \mathbf{J}_{s x}^{m} \\
\mathbf{A}_{x}^{\ell} \mathbf{P}_{z}^{m}+\mathbf{W}_{y}^{\ell} \mathbf{J}_{s y}^{m}
\end{array}\right)
$$

and set

$$
\mathbf{P}_{z}^{m+1}=\mathbf{P}_{z}^{m}+\mathbf{B}_{y}^{\ell} \mathbf{P}_{x}^{m+1 / 2}-\mathbf{B}_{x}^{\ell} \mathbf{P}_{y}^{m+1 / 2}
$$


where

$$
\begin{aligned}
\mathbf{M}_{x}^{\ell}=\Psi_{x}^{\top} \mathbf{W M}_{x} \Psi_{x}, & \mathbf{A}_{E_{x}}^{\ell}=\Psi_{x}^{\top} \mathbf{W} \mathbf{A}_{E_{x}} \Psi_{x}, & \mathbf{A}_{x}^{\ell}=\Psi_{x}^{\top} \mathbf{W} \mathbf{A}_{x} \Psi_{x}, \\
\mathbf{M}_{y}^{\ell}=\Psi_{y}^{\top} \mathbf{W M}_{y} \Psi_{y}, & \mathbf{A}_{E_{y}}^{\ell}=\Psi_{y}^{\top} \mathbf{W} \mathbf{A}_{E_{y}} \Psi_{y}, & \mathbf{A}_{y}^{\ell}=\Psi_{y}^{\top} \mathbf{W} \mathbf{A}_{y} \Psi_{y}, \\
\mathbf{B}_{y}^{\ell}=\Psi_{z}^{\top} \mathbf{W B}_{y} \Psi_{x}, & \mathbf{B}_{x}^{\ell}=\Psi_{z}^{\top} \mathbf{W} \mathbf{B}_{x} \Psi_{y}, & \mathbf{W}_{x}^{\ell}=\Psi_{x}^{\top} \mathbf{W}, \quad \mathbf{W}_{y}^{\ell}=\Psi_{y}^{\top} \mathbf{W},
\end{aligned}
$$

Notice that all matrices in (10) can be computed off-line, i.e., before we start the loop over $m=1$ to $N_{t}$ in (9). Moreover, we have used in (9b) that $\Psi_{z}^{\top} \mathbf{W} \Psi_{z}$ is the identity matrix.

To measure the error in the reduced-order model we introduce the quantity

$$
\operatorname{Err}_{E_{x}}(\ell)=\frac{\sum_{m=0}^{N_{t}} \alpha_{m}\left\|\mathbf{E}_{x}^{m+1 / 2}-\Psi_{x} \mathbf{P}_{x}^{m+1 / 2}\right\|_{\mathbf{W}}^{2}}{\sum_{m=0}^{N_{t}} \alpha_{m}\left\|\mathbf{E}_{x}^{m+1 / 2}\right\|_{\mathbf{W}}^{2}},
$$

where $\alpha_{m}$ 's are the trapezoidal weights for the interval [0,T]. The quantities $\operatorname{Err}_{E_{y}}(\ell)$ and $\operatorname{Err}_{H_{z}}(\ell)$ are def ned in an analogous way.

\section{Remark 4.2}

We mention that one can use a different ansatz for building POD basis as in, e.g. [4]. Hence, one compute the time average of the snapshots of a feld component and subtract it from the corresponding snapshots matrix. We tested this approach too but its performance are similar to the approach presented in the section.

\subsection{Numerical experiments}

In this subsection we present numerical results from different test runs, where we study the performance of our POD Galerkin scheme. The amount of energy captured by an $\ell$-rank basis is $[15,27,35]$

$$
\mathcal{E}(\ell)=\sum_{i=1}^{\ell} \lambda_{i} / \sum_{i=1}^{d} \lambda_{i},
$$

where the $\lambda_{i}$ 's are the eigenvalues satisfying $\lambda_{1} \geq \lambda_{2} \geq \ldots \geq \lambda_{d}>0$. According to this, one can choose the percentage $p$ of energy that the basis should keep and then calculate the minimum amount of vector basis required for that, using the formula

$$
\ell=\operatorname{argmin}\left\{\mathcal{E}(\ell): \mathcal{E}(\ell) \geq \frac{p}{100}\right\} .
$$

We study now the behavior of the POD scheme using two different kind of source waveform and different frequencies. In the following, the medium used for getting the snapshots is the free space.

First, we consider the reduction of two different sinusoidal waves, with different frequencies. We choose for the frst wave $f_{1}=5 \mathrm{kHz}$ and for the second one $f_{2}=20 \mathrm{kHz}$. We consider these 2 simulations on the same space-time grid, so we def ne the grid parameter such that we have stability in the case of the highest frequency, $f_{2}$.

Hence, using $p=99.99 \%$, the results are plotted in Table I. In both cases, we can compute the

\begin{tabular}{ccc} 
f eld variable & sine $f_{1}=5 \mathrm{kHz}$ & sine $f_{2}=20 \mathrm{kHz}$ \\
\hline$E_{x}$ & $\ell_{x}=6$ & $\ell_{x}=22$ \\
$E_{y}$ & $\ell_{y}=6$ & $\ell_{y}=19$ \\
$H_{z}$ & $\ell_{z}=9$ & $\ell_{z}=24$ \\
\hline
\end{tabular}

Table I. Comparison of the number $\ell$ of POD basis needed in the case of sinusoidal waves with different frequencies $(p=99.99 \%)$. 


\begin{tabular}{ccc} 
f eld variable & $\operatorname{sine} f_{1}=5 \mathrm{kHz}$ & sine $f_{2}=20 \mathrm{kHz}$ \\
\hline$E_{x}$ & $\operatorname{Err}_{E_{x}}(6)=3 \cdot 10^{-3}$ & $\operatorname{Err}_{E_{x}}(22)=9 \cdot 10^{-3}$ \\
$E_{y}$ & $\operatorname{Err}_{E_{y}}(6)=3 \cdot 10^{-3}$ & $\operatorname{Err}_{E_{y}}(19)=4 \cdot 10^{-3}$ \\
$H_{z}$ & $\operatorname{Err}_{H_{z}}(9)=1 \cdot 10^{-2}$ & $\operatorname{Err}_{H_{z}}(24)=1 \cdot 10^{-2}$ \\
\hline
\end{tabular}

Table II. Comparison of the POD errors in the case of sinusoidal waves with different frequencies $(p=$ $99.99 \%$ ) using the number of basis vectors in Table I.

POD-error in the reconstruction of the three f elds components, see Table II. We can conclude that for keeping the same amount of energy in the higher frequency case, we need more basis vectors than in the lower case. For every f eld component, in the case of $f_{2}$, we need more than three times the basis number used in the $f_{1}$ case.

In the next simulation we considered two Gaussian pulses: the generic expression is stated in the following equation

$$
s(t)=\exp \left(-\frac{\left(t-t_{0}\right)^{2}}{\tau^{2}}\right),
$$

where $t_{0}=4.5 \tau$ is the time shift used for avoiding starting the waveform in $t=0$ with the maximum value of the pulse and $\tau$ determines the width of the pulse (for more details see [9]). In this case, we do not have a monochromatic source, i.e., we do not have a single frequency. Anyway, we can identify the maximum frequency of the pulse. Hence, we are interested in checking what happens if we choose different maximum frequencies. We use $f_{1 \max }=5 \mathrm{kHz}$ and $f_{2 \max }=10 \mathrm{kHz}$ and we used $f_{2 \max }$ for having the right time step and space step in order to satisfy stability and sampling conditions. The number of POD basis functions are given in Table III. In Table IV we report on

\begin{tabular}{ccc} 
feld variable & Gauss $f_{1 \max }=5 \mathrm{kHz}$ & Gauss $f_{2 \max }=10 \mathrm{kHz}$ \\
\hline$E_{x}$ & $\ell_{x}=2$ & $\ell_{x}=7$ \\
$E_{y}$ & $\ell_{y}=2$ & $\ell_{y}=7$ \\
$H_{z}$ & $\ell_{z}=3$ & $\ell_{z}=8$ \\
\hline
\end{tabular}

Table III. Comparison of the number $\ell$ of POD basis needed in the case of Gaussian pulse waveforms with different maximum frequencies $(p=99.99 \%)$

the POD error for $p=99.99 \%$. As before, we see that if we have a higher maximum frequency, we

\begin{tabular}{ccc} 
f eld variable & Gauss $f_{1 \text { max }}=5 \mathrm{kHz}$ & Gauss $f_{2 \text { max }}=10 \mathrm{kHz}$ \\
\hline$E_{x}$ & $\operatorname{Err}_{E_{x}}(2)=1 \cdot 10^{-3}$ & $\operatorname{Err}_{E_{x}}(7)=1 \cdot 10^{-3}$ \\
$E_{y}$ & $\operatorname{Err}_{E_{y}}(2)=1 \cdot 10^{-3}$ & $\operatorname{Err}_{E_{y}}(7)=2 \cdot 10^{-3}$ \\
$H_{z}$ & $\operatorname{Err}_{H_{z}}(3)=4 \cdot 10^{-3}$ & $\operatorname{Err}_{H_{z}}(8)=1 \cdot 10^{-2}$ \\
\hline
\end{tabular}

Table IV. Comparison of the POD errors in the case of Gaussian pulse waveforms with different maximum frequencies ( $p=99.99 \%$ ) using the number of basis vectors in Table III.

need more basis functions in order to keep the same amount of energy as the lower frequency case. Moreover, the number of POD basis is related to the type of waveform used to having the snapshots. Even having the same frequencies (compare the cases $f_{1}$ for the sinusoidal case and $f_{1 \max }$ for the Gaussian pulse), we need less basis functions in the Gaussian case. Hence, the number of basis functions is inf uenced by the kind of waveform and its frequency.

\section{CONCLUSIONS}

In this paper the problem of determining the EM properties $(\varepsilon(\boldsymbol{x}), \mu(\boldsymbol{x}), \sigma(\boldsymbol{x}))$ of a hidden objects has be considered. This parameter identif cation problem is formulated in terms of a nonlinear 
optimal control problem with Maxwell equations as side constraints. We have discussed the characterization of the solution to the optimization problem as the solution of an optimality system which involves Maxwell equations and adjoint Maxwell equations. Their solution provide the gradient of the reduced cost functional and we have used this gradient to def ne a successful iterative optimization technique to obtain a sequence of approximations of the EM properties. Then, in order to reduce the compuational effort for the solution of the Maxwell equations, we have investigated consider a model reduction technique based on POD. The POD method for different kind of sources and frequencies and we concluded that this technique can catch the behavior of the snapshot with few basis functions.

In the future we would like to use the POD technique for having a basis set for solving the direct problem and another one for the adjoint one. Hence, we should consider the problem of upadting the basis during the iterations of the optimization loop.

\section{REFERENCES}

1. Antoulas A. 2005 Approximation of Large-Scale Dynamical Systems. SIAM, Philadelphia.

2. Bennett C. 1981. Time domain inverse scattering. IEEE Transactions on antennas and propagation, 29:213-219.

3. Berenger J. 1994. A perfectly matched layer for the absorption of electromagnetic waves. Journal of Computational Physics, 114:185-200.

4. Chatterjee A. 2000. An introduction to the proper orthogonal decomposition. Current Science, 78:808-817.

5. Chen Y, Hesthaven J, Maday Y. 2011. A seamless reduced basis element method for 2D Maxwell's problem: an introduction. Spectral and High Order Methods for Partial Differential Equations-Selected papers from the ICASOHOM'09 conference, 76:141-152.

6. Chen Y, Hesthaven J, Maday Y, Rodriguez J, Zhu X. 2011. Certified reduced basis method for electromagnetic scattering and radar cross section estimation. Submitted.

7. Delbary F, Erhard K, Kress R, Potthast R, Schulz J. 2008. Inverse electromagnetic scattering in a two-layered medium with an application to mine detection. Inverse Problems, 24:1-25.

8. Du J, Zhu J, Luo Z, Navon I.M. 2009. An optimizing finite difference scheme based on proper orthogonal decomposition for CVD equations. Communications in Numerical Methods in Engineering, 27:78-94.

9. Elsherbeni A, Demis D. 2009. The Finite-Difference Time-Domain Method for Electromagnetics with Matlab Simulations. Scitech Publishing. Inc., Raleigh.

10. Fares B, Hesthaven J.S., Maday Y, Stamm B. 2011. The reduced basis method for the electric field integral equation. Submitted.

11. Franceschetti G. 1998. Campi elettromagnetici. Bollati Boringhieri, Torino.

12. Ganesh M, Hesthaven J, Stamm B. 2011 A reduced basis method for multiple electromagnetic scattering in three dimensions. Preprint submitted to Journal of Computational Physics.

13. Hinze M, Pinnau R, Ulbrich M, Ulbrich S. 2009. Optimization with PDE Constraints. Springer-Verlag, Berlin.

14. Hinze M, Volkwein S. 2005. Proper orthogonal decomposition surrogate models for nonlinear dynamical systems: error estimates and suboptimal control. In Reduction of Large-Scale Systems, P. Benner, V. Mehrmann, D.C. Sorensen (eds.), Lecture Notes in Computational Engineering, 45:261-306.

15. Holmes P, Lumley J.L., G. Berkooz G. 1996 Turbulence, Coherent Structures, Dynamical Systems and Symmetry. Cambridge Monographs on Mechanics, Cambridge University Press.

16. Ito K, K. Kunisch. 2008. Lagrange Multiplier Approach to Variational Problems and Applications. SIAM, Philadelphia.

17. Jin J. 2010. Theory and Computation of Electromagnetic Fields. Wiley, Hoboken.

18. Kang N, Chung Y, Cheon C, Jung H. 2002. A new 2-D image reconstruction algorithm based on FDTD and design sensitivity analysis. IEEE Transactions on Microwave Theory and Techniques, 50:2734-2740.

19. Kress R. 2009. Inverse scattering theory. Notes Study, Göttingen.

20. Leis R. 1988. Initial Boundary Value Problems in Mathematical Physics. John Wiley, New York.

21. Mancini R. 2009. An Adjoint-Based Optimization Scheme for Solving Time-Domain Electromagnetic Inverse Scattering Problems. Master thesis, Benevento, Italy.

22. Maurer H, Zowe J. 1979. First and second-order necessary and sufficient optimality conditions for infinitedimensional programming problems. Math. Programming, 16:98-110.

23. Monk P, Süli E. 1994. A convergence analysis of Yee's scheme on nonuniform grids. SIAM J. Numer. Anal., 31:393412.

24. Mur G. 1981. Absorbing boundary conditions for the finite-difference approximation of the time-domain electromagnetic-field equations. IEEE Transactions On Electromagnetic Compatibility, 23 (4): 377382.

25. Noack B.R., Morzyński M, Tadmor G. 2011. Reduced-Order Modelling for Flow Control. CISM Courses and Lectures, vol. 528, Springer-Verlag, Wien.

26. Nocedal J, Wright S. 2000. Numerical Optimization. Springer, New York.

27. Pinnau R. 2008. Model reduction via proper orthogonal decomposition. Model Order Reduction: Theory Research Aspects and Applications, Springer Series Mathematics in Industry, Vol. 13.

28. Pozar D. 2005. Microwave Engineering. Wiley, Hoboken.

29. Rekanos I. 2003. Time-domain inverse scattering using Lagrange multipliers: an iterative FDTD-based optimization technique. J. of Electromagn. Waves and Appl., 17:271-289. 
30. Semnani A, Kamyab M. 2007. An enhanced method for inverse scattering problems using fourier series expansion in conjunction with FDTD and PSO. Progress In Electromagnetics Research, 76:45-64.

31. Sun P, Luo Z, Zhou Y. 2010. Some reduced finite difference schemes based on a proper orthogonal decomposition technique for parabolic equations. Applied Numerical Mathematics, 60:154-164.

32. Taf ove A, Hagness S. 2000. Computational Electrodynamics: The Finite-Difference Time-Domain Method. Artech House, Norwood.

33. Tanaka T, Takenaka T, He S. 1999. An FDTD approach to the time-domain inverse scattering problem for an inhomogeneous cylindrical object. Microwave And Optical Technology Letters, 20:72-77.

34. Tröltzsch F. 2010. Optimal Control of Partial Differential Equations. Theory, Methods and Applications. American Mathematical Society, Providence.

35. Volkwein S. 2011. Model Reduction using Proper Orthogonal Decomposition. Lecture Notes, University of Konstanz.

www. math. uni-konstanz.de/numerik/personen/volkwein/teaching/PoD-Vorlesung.pdf

36. Yee K. 1966. Numerical solution of initial boundary value problems involving Maxwell's equations in isotropic media. IEEE Trans. Antennas and Propagation, AP-16:302-307. 